\title{
ESTUDO TEÓRICO DA ESTRUTURA ELETRÔNICA E DA DINÂMICA INDUZIDA POR LASERS DA MOLÉCULA DE HCI
}

\author{
Vinícius V. Cruz e Freddy F. Guimarães* \\ Instituto de Química, Universidade Federal de Goiás, Campus Samambaia, CP 131, 74001-970 Goiânia - GO, Brasil
}

Recebido em 8/6/12; aceito em 5/9/12; publicado na web em 24/1/13

\begin{abstract}
THEORETICAL STUDY OF THE ELECTRONIC STRUCTURE AND THE LASER INDUCED DYNAMICS OF THE HCl MOLECULE. Potential energy and dipole moment curves for the $\mathrm{HCl}$ molecule were computed. Calculations were performed at different levels of theory (DFT, MRCI). Spectroscopic properties are reported and compared with experimental data, for validating the theoretical approaches. Interaction of infrared radiation with $\mathrm{HCl}$ is simulated using the wave packet formalism. The quantum control model for population dynamics of the vibrational levels, based on pi-pulse theory, is applied. The results demonstrate that wavepackets with specific composition can be built with short infrared laser pulses and provide the basis for studies of $\mathrm{H}+\mathrm{HCl}$ collision dynamics with infrared laser excitation.
\end{abstract}

Keywords: $\mathrm{HCl}$; wave packets; short IR laser pulses.

\section{INTRODUÇÃO}

Cálculos de estrutura eletrônica desempenham um papel fundamental dentro do entendimento da reatividade de sistemas atômicos e moleculares. Estudos teóricos, que se baseiam na construção de curvas de potencial, são uma consequência direta da aproximação de Born-Oppenheimer ${ }^{1}$ e permitem a obtenção de diversas propriedades do sistema, como parâmetros espectroscópicos, caracterização de estados de transição, constantes de equilíbrio e superfícies de energia potencial (PES - Potential Energy Surface) . $^{2,3}$ A obtenção de PES, que adequadamente descrevem um sistema molecular, constitui um dos fatores preponderantes nos estudos de dinâmica nuclear. É necessário conhecê-la para tratar a evolução temporal dos núcleos em diversos tipos de simulação computacional, tanto no emprego do formalismo clássico ${ }^{4}$ quanto no do quântico. ${ }^{5-7}$ Em outras palavras, pode-se dizer que o movimento dos núcleos é guiado pela PES.

$\mathrm{Na}$ literatura podem ser encontrados diversos estudos acerca da estrutura eletrônica do $\mathrm{HCl},{ }^{2,3,8}$ incluindo a análise teórico-experimental do espectro Raman ressonante da camada L do átomo de cloro, ${ }^{9}$ assim como do seu momento de dipolo. Por exemplo, na referência 10 a curva do momento de dipolo do $\mathrm{HCl}$ é obtida através de ajustes de dados de espectroscopia rovibracional, nas referências de Buldakov et al. ${ }^{11}$ e Maroulis ${ }^{12}$ métodos semiempíricos e ab initio baseados nas teorias de perturbação de muitos corpos de $4^{\mathrm{a}}$ Ordem e Coupled Cluster $^{1,13}$ são usados na obtenção da curva de energia potencial e do momento de dipolo em função da variação da distância entre os átomos de hidrogênio e cloro.

As teorias da eletrodinâmica quântica (QED - Quantum Electrodynamics) e da aproximação semiclássica são, geralmente, empregadas em estudos teóricos relativos à interação luz-matéria. No tratamento teórico através da eletrodinâmica quântica considera-se que o problema está sendo resolvido de maneira exata, o caráter discreto tanto da radiação quanto da matéria são levados em conta. Já na aproximação semiclássica somente a matéria é considerada ter natureza quântica sendo descrita pela equação de Schrödinger, enquanto a radiação é considerada clássica e, deste modo, modelada pelas equações de Maxwell do eletromagnetismo. Este tipo de aproximação é razoável para pulsos intensos e de baixa frequência, onde o aspecto descontínuo

*e-mail: freddy@quimica.ufg.br da radiação pode ser negligenciado. ${ }^{14}$ A partir da obtenção da PES e utilizando a aproximação semiclássica é possível aplicar o formalismo de pacotes de ondas para tratar o sistema molecular na presença de pulsos de laser. Neste caso, pode ser feita a solução numérica da equação de Schrödinger dependente do tempo na presença de uma radiação eletromagnética coerente. Soluções analíticas também são possíveis para sistemas idealizados. A solução de Rabi para sistemas de dois níveis leva à obtenção da teoria de pulso $\pi,{ }^{15}$ que mostra que a composição final de uma superposição coerente de estados quânticos (CSQS - Coherent Superposition of Quantum States), de um sistema que interage com um laser, está diretamente relacionada com a área do pulso. Esta teoria mostra a possibilidade de propor modelos de controle de população para sistemas restritos a dois níveis vibracionais.

Neste artigo, estudou-se a molécula de $\mathrm{HCl}$ através de cálculos de estrutura eletrônica e simulações computacionais de sua interação com a radiação eletromagnética na região do infravermelho. Informações a respeito do cloreto de hidrogênio são relevantes, devido à sua simplicidade e importância ambiental na química atmosférica. $\mathrm{O} \mathrm{HCl}$ reage com diversos compostos gerando o radical $\mathrm{Cl}$, que é uma das espécies que contribuem para a destruição da camada de ozônio. ${ }^{16}$ Os resultados dos cálculos de estrutura eletrônica apresentados são baseados na teoria do funcional de densidade (DFT - Density Functional Theory $)^{1}$ e no método de interação configuracional multirreferencial (MRCI-Multi-Reference Configuration Interaction) ${ }^{1,13}$ São computadas as curvas de energia potencial, momento de dipolo e a variação do momento de dipolo em relação às distâncias interatômicas. A simulação da interação da molécula de $\mathrm{HCl}$ com pulsos curtos de laser é feita através do formalismo de pacotes de onda, ${ }^{5,6,17}$ onde são aplicadas as curvas de potencial e a variação do momento dipolo obtidas pelos cálculos de estrutura eletrônica.

\section{PARTE TEÓRICA}

\section{Cálculos de estrutura eletrônica}

Todos os cálculos de estrutura eletrônica apresentados neste artigo foram realizados com o pacote computacional de química quântica GAMESS, ${ }^{18}$ onde os métodos DFT e MRCI são empregados na construção das curvas de energia potencial e de momento de dipolo. Os resultados dos cálculos DFT são obtidos a partir de uma referência 
restrita (RDFT) e não restrita (UDFT) utilizando o funcional híbrido X3LYP. ${ }^{19}$ As configurações iniciais dos cálculos MRCI são obtidas por um cálculo de interação multiconfiguracional ${ }^{1,13}$ (MCSCF Multiconfiguration Self Consistent Field) em um espaço ativo completo (CAS - Complete Active Space) com 6 elétrons ativos em 11 orbitais. É apresentada uma comparação entre os resultados RDFT ${ }^{1}$, $\mathrm{UDFT}^{1}$ e MRCI ${ }^{1}$ com dados da literatura, ${ }^{20}$ demonstrando os pontos fortes e as deficiências das técnicas utilizadas. Os seguintes conjuntos de bases atômicas são utilizados nos cálculos: aug-cc-pVDZ, aug-cc-pVTZ e aug-cc-pVQZ. A aplicação dos métodos e dos conjuntos de bases descrita acima levou à construção de 9 curvas de energia potencial e de momento de dipolo para a variação interatômica dos átomos da molécula de $\mathrm{HCl}$.

Com as curvas de energia potencial da molécula de $\mathrm{HCl}$, as autofunções e autoenergias do sistema foram computadas com o programa eSPec. ${ }^{21}$ Através de uma parametrização baseada no potencial de Morse $^{20,22}$ foi possível calcular os parâmetros espectroscópicos do HCl. Os parâmetros espectroscópicos calculados são confrontados com dados experimentais, ${ }^{20}$ para comparação da qualidade dos resultados obtidos pelos métodos teóricos empregados neste artigo.

\section{Controle de população com laser}

O controle quântico de reações químicas pode, por exemplo, ser feito através da preparação de superposições de níveis vibracionais da molécula de $\mathrm{HCl}$ antes dela colidir com um átomo, íon ou molécula. Estudos teóricos de população de níveis vibracionais são, em geral, feitos pela resolução numérica da equação de Schrödinger dependente do tempo sob a ação de um campo eletromagnético externo. ${ }^{23}$ Quando um pulso de laser ressonante atinge o sistema molecular ocorre a formação de CSQS que podem ser escritas matematicamente na forma:

$$
\Psi(t)=\sum_{i} C_{i(t)} \psi_{i}
$$

onde $\psi_{i}$ é a função de onda do $i$-ésimo nível vibracional. A probabilidade $P_{i}$ de o sistema estar em um dos respectivos níveis vibracionais é dada pelo módulo quadrado dos coeficientes $C_{i}$ :

$$
P_{i}=\left|C_{i(t)}\right|^{2}=\left|\left\langle\Psi(\mathrm{t}) \mid \psi_{i}\right\rangle\right|^{2} .
$$

Nas simulações computacionais aqui apresentadas é considerado um pulso de laser, para preparar as CSQS, descrito pela Equação 3:

$$
E(t)=\sqrt{\frac{2 I(t)}{c \epsilon_{0}}} \cos (\omega \mathrm{t}+\eta)
$$

onde $\omega$ é a frequência da radiação, $\eta$ é a fase inicial da onda e $I(t)$ é a função intensidade, que determina o formato do pulso, a qual é modelada por:

$$
I(t)=I_{0} \exp \left[-\ln 2\left(\frac{t-t_{0}}{\tau}\right)^{2 m}\right]
$$

Esta forma para a intensidade leva a uma transição suave de um pulso gaussiano ( $m=1$ a um pulso retangular $(m \rightarrow \infty)$, com intensidade máxima $I_{0}$, onde $\tau$ é a meia largura a meia altura (HWHM) e $t_{0}$ o tempo em que a interação atinge o valor máximo. Nas simulações computacionais realizadas é considerado que o laser aplicado está alinhado com a coordenada internuclear da molécula.

Para o caso restrito de dois estados quânticos, a probabilidade de popular os estados $i=0$ e 1, é dada pela solução analítica de Rabi,

$$
P_{0}=\cos ^{2}\left(\frac{\theta(\mathrm{t})}{2}\right), P_{1}=\sin ^{2}\left(\frac{\theta(\mathrm{t})}{2}\right)
$$

onde se observa que a dependência na probabilidade de popular os estados quânticos está relacionada com a área do pulso $\omega(\mathrm{t})$ aplicado ao sistema. ${ }^{15,23} \mathrm{~A}$ área de um pulso, descrito pela Equação 4, para um tempo infinito, é dada por: ${ }^{23}$

$$
G_{(\infty)}=\frac{G_{0} \tau}{m}\left(\frac{2}{\ln 2}\right)^{\frac{1}{2}} \Gamma\left(\frac{1}{2 m}\right)
$$

onde a frequência de Rabi do sistema $G_{0}=E_{0} d_{01} / \hbar$, com d $\mathrm{d}_{01}$ sendo o momento de dipolo de transição, $E_{0}$ a energia máxima do pulso de laser e $\Gamma(\mathrm{x})$ a função gama, ${ }^{24}$ a qual é uma extensão da função fatorial subtraída de 1. Resolvendo as Equações 5 e 6 pode-se obter o tempo de duração (HWHM) do pulso que se deve aplicar sobre o sistema, restrito a dois estados, para obter uma determinada composição do pacote de ondas final. Este tipo de análise é conhecido como teoria de pulsos $\pi$. Com o objetivo de testar a teoria de pulsos $\pi$ em sistemas constituintes de vários estados quânticos, foram feitas simulações numéricas considerando laser com diversos tempos de duração, na busca de um regime que minimizasse a população do terceiro nível vibracional, assim como dos níveis de maiores energias.

\section{RESULTADOS E DISCUSSÃO}

A Figura 1 apresenta as curvas de energia potencial para a molécula de $\mathrm{HCl}$, calculadas com a base aug-cc-pVTZ, e autofunções para os 6 primeiros níveis vibracionais $v_{j} \operatorname{com} j=0,1,2,3,4$ e 5, as quais foram trasladadas para os seus respectivos autovalores a título de visualização. Como pode ser observado na Figura 1, os cálculos restritos levam a uma superestimação da energia de dissociação do sistema, este resultado é conhecido e bem descrito na literatura, ${ }^{1,13}$ pois neste método os elétrons se agrupam em pares não sendo permitida a ocupação simples dos orbitais moleculares. Curvas de energia potencial provenientes de cálculos restritos representam a quebra heterolítica da ligação química, levando à formação de fragmentos iônicos. A quebra homolítica da ligação química é adequadamente representada por cálculos não restritos, que permitem a ocupação simples de orbitais moleculares e formação de espécies radicalares. Deste modo, observa-se que cálculos restritos fornecem resultados razoáveis apenas próximos da distância de equilíbrio para estudos considerando as moléculas no vácuo. Portanto, os resultados das energias de dissociação $\mathrm{D}_{0}$ dos cálculos UDFT e MRCI fornecem valores mais próximos em relação aos dados experimentais. Isto

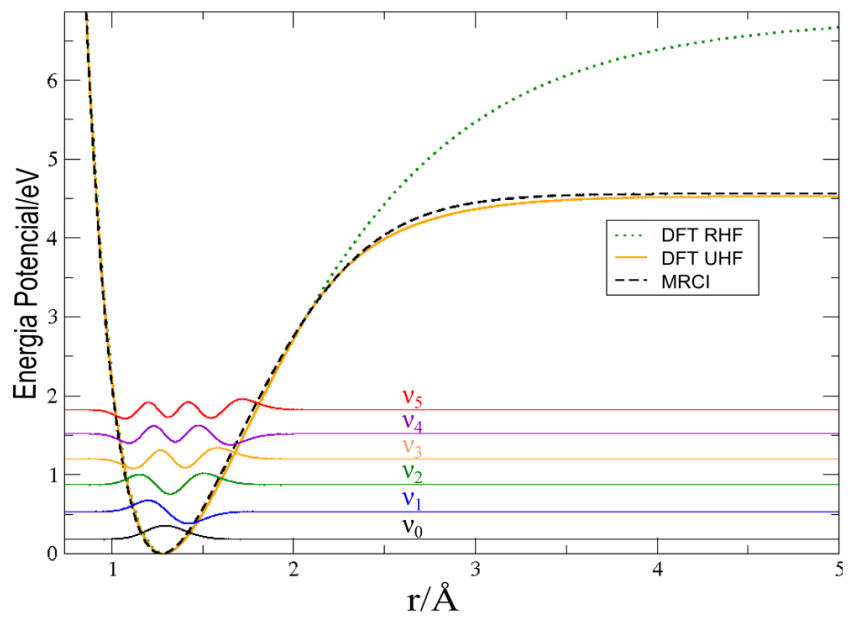

Figura 1. Curvas de energia potencial para a molécula de $\mathrm{HCl}$ em diferentes níveis de teoria na base aug-cc-pVTZ e as 6 primeiras autofunções vibracionais 
pode ser visto através da comparação dos resultados de energia de dissociação obtidos pelos cálculos de estrutura eletrônica e dados experimentais apresentados na Tabela 1.

Tabela 1. Parâmetros espectroscópicos, distância de equilíbrio $\mathrm{r}_{\mathrm{e}}$, frequência harmônica $\omega_{\mathrm{e}}$, constante de anarmônicidade $\omega_{\mathrm{e}} \mathrm{x}_{\mathrm{e}}$ e energia de dissociação $\mathrm{D}_{0}$, para a molécula de $\mathrm{HCl}$

\begin{tabular}{llcccc}
\hline & Base & $\mathrm{r}_{\mathrm{e}} / \AA$ & $\omega_{\mathrm{e}} / \mathrm{cm}^{-1}$ & $\omega_{\mathrm{e}} \mathrm{x}_{\mathrm{e}} / \mathrm{cm}^{-1}$ & $\mathrm{D}_{0} / \mathrm{eV}$ \\
\hline \multirow{4}{*}{ UDFT } & aug-cc-pVDZ & 1,289 & 2963,907 & 46,563 & 4,793 \\
& aug-cc-pVTZ & 1,285 & 2932,997 & 48,328 & 4,351 \\
& aug-cc-pVQZ & 1281 & 2931,658 & 47,900 & 4,370 \\
\hline \multirow{4}{*}{ MRCI } & aug-cc-pVDZ & 1,285 & 2988,465 & 51,913 & 4,285 \\
& aug-cc-pVTZ & 1,274 & 3010,365 & 51,687 & 4,378 \\
& aug-cc-pVQZ & 1,272 & 3013,257 & 51,362 & 4,396 \\
& Experimental $^{19}$ & 1,275 & 2989,740 & 52,050 & 4,430 \\
\hline
\end{tabular}

Na Tabela 1, além dos dados de $\mathrm{D}_{0}$, são apresentados também os resultados relativos aos parâmetros espectroscópicos, calculados segundo a parametrização do potencial de Morse, juntamente com valores experimentais. ${ }^{20}$ Uma análise comparativa dos dados da Tabela 1 mostra que os resultados obtidos a partir dos cálculos MRCI estão mais próximos dos valores experimentais que os DFT. Este resultado indica uma superioridade das curvas de energia potencial MRCI em relação às DFT. Pode ser observado, também, que não existe uma melhora consistente dos resultados em relação à qualidade da base utilizada no cálculo, apesar de terem sido utilizados nos cálculos conjunto de bases de correlação consistente.

As curvas de momento de dipolo calculadas são mostradas na Figura 2. Apesar das curvas de energia potencial em UDFT e MRCI serem, em geral, próximas, o mesmo não foi observado para as curvas de momento de dipolo, onde as curvas MRCI decaem a zero mais rapidamente com a separação internuclear do que as curvas UDFT. À primeira vista, tem-se a impressão que as curvas de momento de dipolo dos resultados DFT e MRCI são semelhantes, em torno da região de equilíbrio. Entretanto, uma análise mais criteriosa, a partir das variações das curvas de momento de dipolo, mostra que existe uma discrepância qualitativa entre os resultados DFT e MRCI, que vai além de um simples deslocamento de um valor numérico de uma curva em relação à outra. Para melhor visualização da discussão são apresentadas na Figura 3 as curvas de momento de dipolo na região de equilíbrio no painel superior, no painel do meio as derivadas em relação à distância interatômica do momento de dipolo, e a função de onda do nível vibracional zero no painel inferior. Foram omitidas na Figura 3 as curvas correspondentes à base aug-cc-pVTZ, devido a sua similaridade com as curvas aug-cc-pVQZ. A variação das curvas de momento de dipolo, que é fator preponderante para absorção de energia vibracional na região do infravermelho, mostra que as derivadas dos momentos de dipolo dos cálculos DFT e MRCI apresentam discrepâncias qualitativas para distâncias de ligação maiores que 2,75 Bohr. De acordo com o painel inferior da Figura 3, uma parte da função de onda é diretamente afetada pelas variações distintas do momento de dipolo durante a interação com a radiação eletromagnética. Isto indica que simulações computacionais baseadas em pacotes de onda devem ser sensíveis a esta diferença.

Para efeito de comparação, são apresentadas na Figura 4 as curvas apresentadas neste artigo e as descritas na literatura. $\mathrm{O}$ modelo gerado por Li e colaboradore ${ }^{10}$ é obtido a partir do ajuste de dados experimentais de espectros rovibracionais do $\mathrm{HCl}$, onde são incluídas transições entre os níveis vibracionais $\mathrm{D} v=v_{j}-v_{0}$ com nível variando de 1-7. Neste tipo de aproximação, obtém-se uma

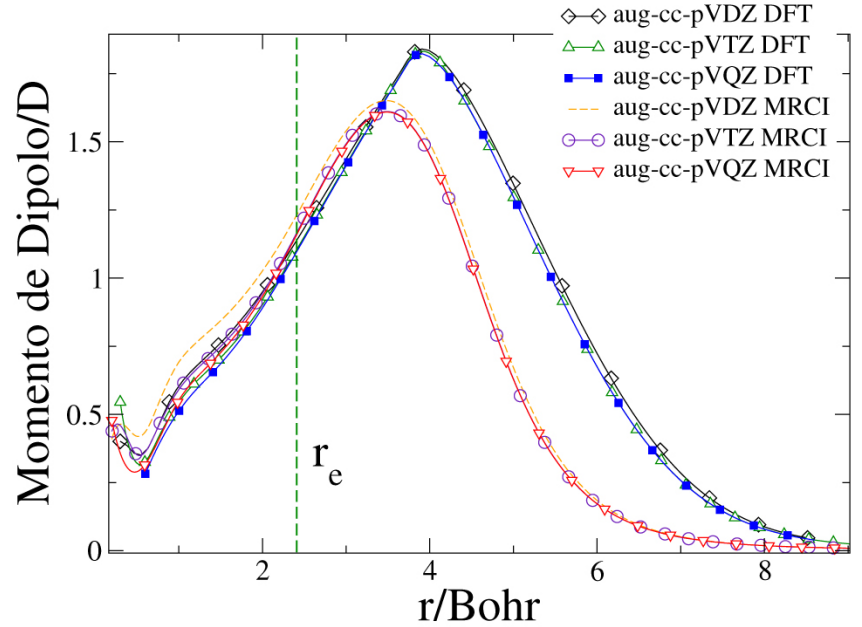

Figura 2. Curvas de momento de dipolo para a molécula de $\mathrm{HCl}$ em função da distância interatômica em diferentes níveis de teoria nas bases aug-cc$-p \operatorname{VnZ}(n=D, T, Q)$
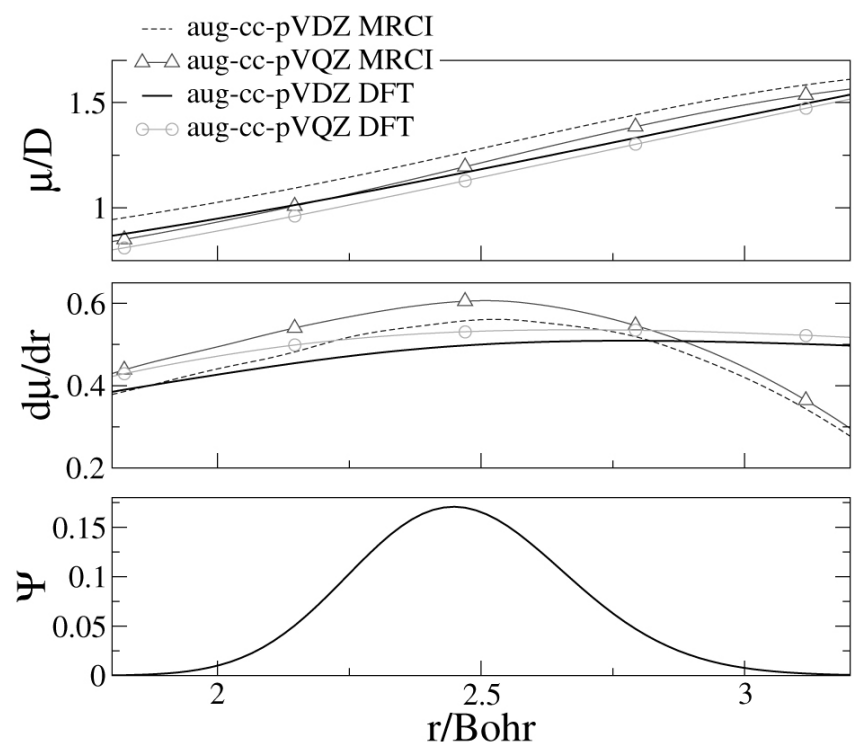

Figura 3. Curvas de momento de dipolo, variação do momento de dipolo e a autofunção vibracional para o estado em função da distância de ligação interatômica do $\mathrm{HCl}$, ampliadas próximo à região de equilíbrio

curva de momento de dipolo que é válida somente no intervalo de distância interatômicas entre 0,986 a 1,925 ̊ (1,861-3,633 Bohr). Isto é uma limitação deste modelo para estudos próximos à região de dissociação. Portanto, o ajuste de Li e colaboradore ${ }^{10}$ só é adequado à descrição de processos em que a função de onda molecular acesse distâncias de ligação menores que $1,93 \AA$. Como um dos interesses deste trabalho é obter curvas de momentos de dipolos que permitam simular computacionalmente a interação da molécula de $\mathrm{HCl}$ com lasers de alta intensidade, onde o pacote de onda visita regiões do sistema molecular próximo à região de dissociação, torna-se importante conhecer o comportamento adequado da curva de momento de dipolo para distâncias de ligação maiores que $2 \AA$. A análise das curvas de momento de dipolo apresentadas na Figura 4 mostra que os resultados dos cálculos MRCI e UDFT têm comportamento adequado na região de dissociação, onde se espera que o momento de dipolo seja nulo para dissociações homolíticas. As comparações dos resultados dos cálculos teóricos feitos neste trabalho com os experimentais indicam que a variação do momento de dipolo obtido pelo MRCI está mais 
próxima dos dados do ajuste experimental de Li et al..$^{10}$ do que os cálculos UDFT e o modelo usado por Kiriyama e colaboradores. ${ }^{25}$ Este resultado pode ser melhor visualizado na Figura 4, que mostra que a variação do momento de dipolo da curva calculada em DFT se aproxima do ajuste experimental somente para valores no entorno da distância de equilíbrio $(0,986-1,455 \AA$ ̊ ) divergindo à medida que os núcleos se afastam, e que a variação da curva MRCI apresenta comportamento semelhante ao observado para o ajuste experimental. Desta maneira, pode-se considerar que as curvas de energia eletrônica e momento de dipolo calculadas pelo método MRCI consistem em um modelo mais adequado para as simulações computacionais da interação da radiação IR com a molécula de $\mathrm{HCl}$.

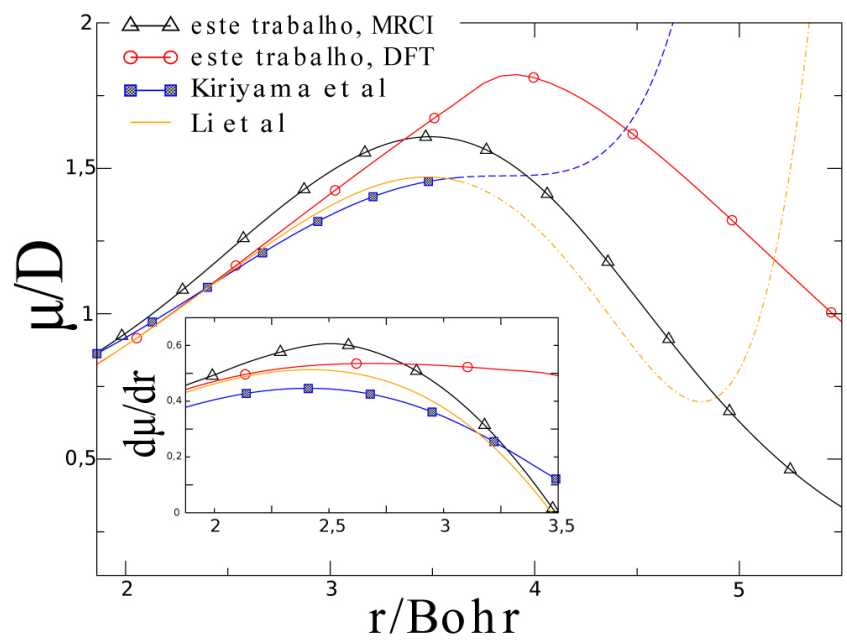

Figura 4. Curvas de momento de dipolo calculadas na base aug-cc-pVQZ e presentes na literatura, em destaque são mostradas as variações do momento de dipolo das respectivas curvas para efeito de comparação

Com as curvas calculadas foram realizadas simulações computacionais de controle de população de estados vibracionais da molécula de $\mathrm{HCl}$ empregando o programa eSPec. Um exemplo deste tipo de dinâmica pode ser apreciado na Figura 5, onde a molécula inicialmente no nível vibracional fundamental $v_{0}$ interage com o laser. A dinâmica de população da Figura 5 mostra inicialmente a diminuição da população do nível $v_{0}$ e o aumento da população do primeiro nível vibracional excitado $v_{1}$ à medida que o laser interage com o sistema ocorre a inversão total da população no tempo $t=9,3$ ps e da reinversão da população no em $t=12,2$ ps. A diferença entre estes tempos é metade do período de Rabi. Continuando a análise observa-se em seguida que o nível $v_{0}$ volta ser depopulado até finalmente o laser deixar de interagir com o sistema, ocorrendo a estabilização das populações dos níveis 0 e 1 em 50:50. Nesta simulação, foi aplicado um laser com parâmetros $I_{0}=2,5.10^{10} \mathrm{~W} / \mathrm{cm}^{2}$, e $\omega=2 \pi\left(v_{0}-v_{1}\right)=$ $0,357 \mathrm{eV}$, com o intuito de minimizar a população do terceiro nível vibracional (utilização de intensidades moderadas), de forma que as probabilidades de população $P_{0}$ e $P_{1}$ se aproximassem da solução analítica da teoria de pulsos $\pi$, Equação 5 .

As simulações realizadas para diversos tempos de duraçãodos pulsos de laser fornecem a frequência de Rabi do sistema $G_{0}=1,142 \cdot 10^{12} \mathrm{rad} / \mathrm{s}$. Através da Equação 6 é construída a curva da variação da população dos níveis vibracionais com o tempo de duração do pulso (área) prevista pelo modelo de pulsos $\pi$, que é comparada com os resultados da simulações numéricas baseada na solução da Equação de Schrödinger dependente do tempo para um sistema de vários níveis (Figura 6). Pode-se observar uma boa concordância entre as simulações numéricas e a curva do modelo analítico, as pequenas diferenças se devem a uma pequena população

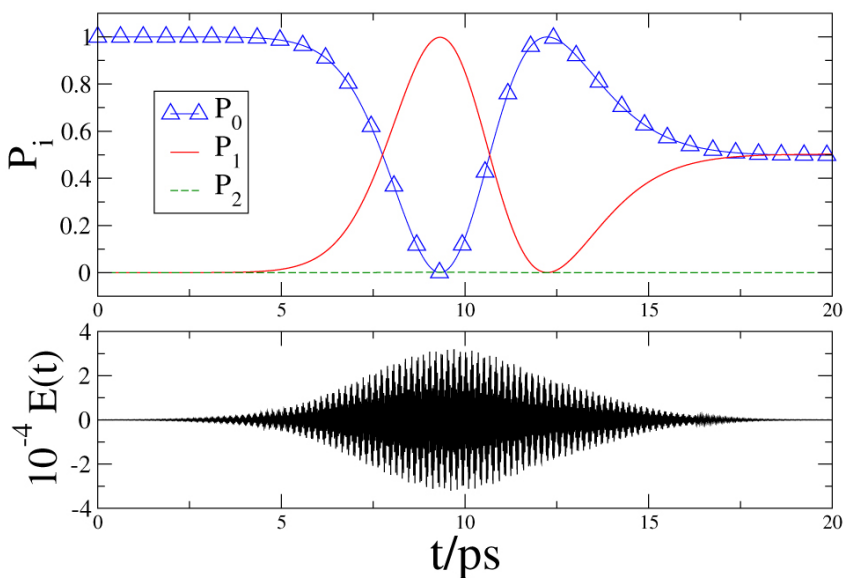

Figura 5. No painel superior é apresentada a dinâmica de população dos estados vibracionais da molécula de HCl. No painel inferior tem-se a representação da variação no tempo da forma do pulso eletromagnético. Neste cálculo foi empregada a curva de dipolo calculada em MRCI(aug-cc-pVQZ), partindo do estado $v_{\underline{0}}$, com um laser com $I_{0}=2,5.10^{10} \mathrm{~W} / \mathrm{cm}^{2}, \tau=2,219 \mathrm{ps}, e$ frequência de ressonância da transição 0-1 do $\mathrm{HCl}$, as composições finais do pacote de ondas correspondem a $P_{0}(\infty)=50 \%$ e $P_{1}(\infty)=50 \%$

do segundo nível vibracional excitado $v_{2}$, durante a interação da

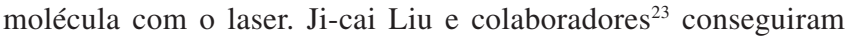
reproduzir esta solução para a molécula de NO regulando minuciosamente a intensidade do laser, evitando qualquer transição para $v_{2}$ durante todo o tempo de interação do laser com a molécula. Através da Figura 6 obtém-se o tempo de pulso necessário para se construir uma determinada superposição coerente entre os níveis $v_{0}$ e $v_{1}$. Este resultado é importante quando se considera a preparação de estados vibracionais, no controle quântico dos produtos de espalhamentos atômico-moleculares, onde é necessário construir superposições coerentes de estados quânticos específicas.

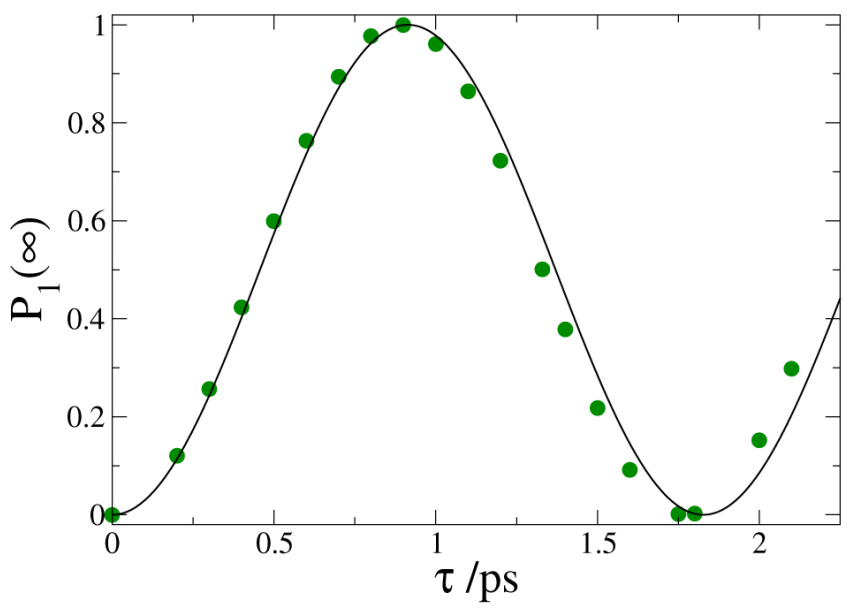

Figura 6. População do primeiro estado vibracional dado pela dinâmica exata, círculos, e a solução analítica para um sistema restrito a dois estados, curva sólida. Os parâmetros utilizados para o laser são os mesmos descritos nas condições da Figura 4 para diferentes tempos de duração

\section{CONCLUSÃO}

A análise dos resultados obtidos permite sumarizar que os cálculos DFT fornecem energias razoáveis em relação aos cálculos MRCI (mais robustos) nas regiões de equilíbrio. Resultados comparáveis aos dados experimentais são obtidos para as constantes espectroscópicas para as curvas de energia potencial por ambos os métodos de cálculo 
empregados neste trabalho. Em relação aos momentos de dipolo, observa-se uma discrepância entre os resultados utilizando níveis de cálculo DFT, MRCI e os dados experimentais, principalmente quando são consideradas para distâncias de ligação maiores que 2 Å. A curva da variação do momento de dipolo obtida com o cálculo MRCI apresenta um comportamento similar aos obtidos por curvas obtidas por ajustes de dados experimentais na região de equilíbrio. Além disto, ela se comporta adequadamente na região de dissociação, onde é esperado um momento de dipolo nulo.

A comparação dos resultados da solução analítica de Rabi para dois estados e as soluções numéricas de pacote de onda, onde estão presentes todos os níveis vibracionais, para população de estados vibracionais mostrou uma concordância qualitativa excelente. Do ponto de vista quantitativo, observa-se apenas um pequeno deslocamento entre a curva analítica e a simulação numérica de pacotes de onda. Deste modo, pode-se concluir que o uso de lasers com intensidades e tempos de duração adequados, que restrinjam o sistema de vários níveis a se comportar como um sistema de dois estados, permite a construção de pacotes de onda com qualquer composição desejada. Para manter a dinâmica de população restrita a dois níveis é necessária regulagem adequada da intensidade e do tempo de duração do pulso de laser.

\section{AGRADECIMENTOS}

Ao CNPq, CAPES e FUNAPE-UFG pelo apoio financeiro utilizado na criação e consolidação do Laboratory of Theoretical and Computational Chemistry (LTCC) da UFG. Ao Dr. I. Rodrigues por estimulantes discussões acerca de controle de reações químicas. V. V. Cruz agradece também ao CNPq por sua bolsa de estudos (PIBIC).

\section{REFERENCIAS}

1. Jensen, F.; Introduction to Computational Chemistry, $2^{\text {nd }}$ ed., Wiley: Chichester, 2006.

2. Allison, T. C.; Lynch, G. C.; Truhlar, D. G.; Gordon, M. S.; J. Phys. Chem. 1996, 100, 13575.

3. Bian, W.; Werner, H.; J. Chem. Phys. 2000, 112, 220.

4. Tachicawa, H.; J. Phys. Chem. A 2002, 106, 6915.

5. McCullough, E. A.; Wyatt, R. E.; J. Chem. Phys. 1970, 54, 3578.
6. Kulander, K. C.; J. Chem. Phys. 1978, 69, 5064.

7. Guimaraes, F. F.; Kimberg, Gelmukhanov, F.; Ågren, H.; Phys. Rev. A 2004, 70, 62504.

8. Xiao-Niu, Z.; De-Heng, S.; Jin-Ping, Z.; Zun-Lüe, Z.; Jin-Feng, S.; Chin. Phys. B 2010, 5, 53401 .

9. Såthe, C.; Guimarães, F. F.; Rubenson, J.-E.; Nordgren, J.; Agui, A.; Guo, J.; Ekström, U.; Norman, P.; Gelmukhanov, F.; Ågren, H.; Phys. Rev. A 2006, 74, 912 .

10. Li, G.; Gordon, I. E.; Bernath, P. F.; Rothman, L. S.; J. Quant. Spectrosc. Radiat. Transfer 2011, 112, 1543.

11. Buldakov, M. A.; Cherepanov, V. N.; J. Phys. B: At. Mol. Opt. Phys. 2004, 37, 3973.

12. Maroulis, G.; J. Chem Phys. 1998, 108, 5432.

13. Szabo, A.; Ostlung, N. S.; Modern Quantum Chemistry - Introduction to advanced Electronic Structure Theory, Dover: New York, 1989.

14. Messiah, A.; Quantum Mechanics, Dover: New York, 1999.

15. Holthaus, M.; Just, B.; Phys. Rev. A 1994, 49, 1950.

16. Wayne, R. P.; Chemistry of Atmospheres, Oxford University: Oxford, 1991.

17. Guimaraes, F. F.; Kimberg, V.; Felicissimo, V. C.; Gelmukhanov, F.; Cesar, A.; Ågren, H.; Phys. Rev. A 2005, 71, 043407.

18. Schmidt, M. W.; Baldridge, K. K.; Boatz, J. A.; Elbert, S. T.; Gordon, M. S.; Jensen, J. H.; Koseki, S.; Matsunaga, N.; Nguyen, K. A.; Su, S. J.; Windus, T. L.; Dupuis, M.; Montgomery, J. A.; J. Comp. Chem. 1993, $14,1347$.

19. Xu, X.; Goddard III, W. A.; Proc. Natl. Acad. Sci. U.S.A. 2004, 101, 2673.

20. Herzberg, G. F. R. S.; Molecular Spectra And Molecular Structure I Spectra Of Diatomic Molecules, Van Nostrand: New York, 1950.

21. Guimarães, F. F.; Programa para resolução numérica da Equação de Schrödinger dependente do tempo (2004), pode ser obtido entrando em contato com o autor principal no e-mail: freddy@quimica.ufg.br.

22. Morse, P. M.; Phys. Rev. 1929, 34, 57.

23. Liu, J.; Felicíssimo, V. C.; Guimarães, F. F.; Wang, C.; Gel'mukhanov, F.; J. Phys. B 2008, 41.

24. Boas, M. L.; Mathematical Methods in the Physical Sciences, $3^{\text {rd }}$ ed.,Wiley: Hoboken, 2006.

25. Kiriyama, F.; Rao, B. S.; Nangia, V. K.; J. Quant. Spectrosc. Radiat. Transfer 2001, 69, 35. 